\title{
Una revisión de diseños longitudinales para estudiar el burnout académico (2010 -2016)
}

\section{A review of longitudinal designs for studying academic burnout (2010-2016)}

\author{
Jorge Enrique Palacio-Sañudo ${ }^{1}$ Ph.D; Carmen Cecilia Caballero-Domínguez² Ph.D; \\ Yuly Paola Suárez-Colorado ${ }^{3}$ M.Sc.
}

\begin{abstract}
${ }^{1}$ Universidad del Norte, Colombia. E-mail: jpalacio@uninorte.edu.co. ${ }^{2}$ Universidad del Magdalena, Colombia. E-mail: ccaballero@unimagdalena.edu.co. ${ }^{3}$ Universidad del Magdalena, Colombia. E.mail: ysuarez@unimagdalena.edu.co.
\end{abstract}

Recibido: 02-04-2017; Aceptado: 16-06-2017

\section{Resumen}

Existe limitada evidencia acerca del estudio longitudinal del burnout académico, sin embargo, este diseño de investigación es necesario para profundizar en la naturaleza del síndrome, en términos de establecimiento, desarrollo y extinción. A través de un estudio bibliométrico, descriptivo-retrospectivo, se realizó una revisión de estudios longitudinales entre 2010-2106, para estudiar el burnout en estudiantes de secundaria y universitarios. Se indagó en las bases de datos Science Direct, Scopus y PubMed, con palabras claves como: academic, burnout, longitudinal. De un universo de 4655 artículos, se identifican 15, pero solo siete están en acceso abierto, y se describen. Los resultados muestran que los diseños longitudinales se han publicado más en la revista Med Teach y los autores que más utilizan este diseño son Noh, Lee, Tartas, Walkiewiichz, Majkowicz, durante los años 2012 y 2015, en muestras de Korea y Estados Unidos. El Inventario de Burnout en la Escuela fue el instrumento más utilizado. Los resultados presentan un síndrome que se incrementa con el tiempo, pero puede reducirse con intervención clínica.

Palabras clave: Burnout, estudiantes, estudio longitudinal, salud mental. 
Palacio-Sañudo et al - Una revisión de diseño longitudinales para estudiar el burnout

\section{Abstract}

There is limited evidence about the longitudinal study of academic burnout, research design necessary to deepen the nature of the syndrome, in terms of establishment, development and extinction. Through a bibliometric study, descriptive-retrospective, a review of longitudinal studies was carried out between 2010-2106, to study burnout in secondary and university students. It asked in the Databases Science direct, Scopus and PubMed, with key words such as: academic, burnout, longitudinal. Of a universe of 4655 articles, they identify 15, but only seven are in open access, and are described. The results show that the longitudinal designs have been published more in the magazine Med teach, and the authors who use this design most are Noh, Lee, tarts, Walkiewiichz, Majkowicz, during the years 2012 and 2015, in samples of Korea and the United States. The burnout inventory at school was the most widely used instrument. The results show a syndrome that increases over time, but can be reduced with clinical intervention.

Keywords: Burnout, students, longitudinal study, mental health.

\section{Introducción}

El estudio del burnout se ha extendido al ámbito académico consolidándose como un estado mental negativo de cansancio físico-emotivo, apatía e ideas de incompetencia relacionado con el rol del estudiante, ocupaciones académicas y dinámicas del contexto universitario; surge en consecuencia y respuesta al estrés crónico y se convierte en uno de los responsables de la insatisfacción y falta de compromiso con la formación académica (Schaufeli, Salanova, González-Romá \& Bakker, 2002; Salanova, Grau, Martínez \& Llorens, 2004; Bresó \& Salanova, 2005; Caballero \& Bresó, 2015).

La naturaleza del burnout académico se caracteriza por su evolución negativa que llega a afectar el bienestar de los estudiantes (Bresó, 2008; Caballero, Palacio y Hederich, 2010), sin embargo, son pocos los estudios longitudinales en contexto académico que permiten verificar la evolución de este proceso, por lo cual es necesario realizar una revisión de las investigaciones más actuales que tengan en cuenta este aspecto. Los estudios trasversales han sido los más numerosos y han contribuido a entender su naturaleza, pero la exploración longitudinal proporcionará mayores insumos para conocer su establecimiento, desarrollo y extinción.

En la actualidad, es ampliamente conocido que el "burnout" es producto del estrés crónico generado por la continua exposición a los factores de riesgo psicosocial (Yan y Tang, 2003), y que requiere un tiempo prologando para el establecimiento de los síntomas emocionales, cognitivos, físicos y conductuales (Salanova, Martínez \& Lorente, 2005; Parraga, 2005; GilMonte \& García Juesas, 2008), los cuales se hacen evidentes de manera progresiva, haciendo que el síndrome vaya ganando fuerza en el sujeto, y de allí la dificultad para su diagnóstico.

Por esto el síndrome de burnout no puede ser visto como un estado estático, sino que presenta cambios en su severidad, que se pueden observar durante diferentes tiempos, tal como un semestre académico, en la transición de un año escolar a otro, e incluso durante la carrera universitaria. Algunos estudios con diseños longitudinales sobre burnout en distintas poblaciones confirman esta evolución en observaciones que van de seis meses a más de dos años, tal como se describen a continuación: 
Seis meses: Se encontraron dos estudios, el primero de Salanova, Martínez y Llorente (2005), quienes evaluaron docentes en dos momentos con una diferencia de seis meses, y encontraron un incremento en las dimensiones de agotamiento, cinismo al inicio y final de un curso académico pero también encontraron bajos niveles de despersonalización. El segundo de Park y Lee (2013), quienes evaluaron el "burnout" en tres momentos en los profesores de secundaria y preparatoria. Los resultados indicaron cambios en el agotamiento emocional entre el primer y segundo seguimiento, de despersonalización entre el segundo y tercero, además de cambios de realización personal entre la primera y segunda medición.

Un año: Se identificaron cuatro estudios, el primero de Wade, Coolery y Savicki (1986), quienes registraron los niveles de "burnout" en profesionales de ayuda, se halló que eran bastante estables a lo largo de un año. Del mismo modo, Llorens, García-Renedo y Salavoa (2005), encontraron estos cambios en el "burnout" en observaciones de dos tiempos con un intervalo de un año entre ellas. El tercero es de GrauAlberola, Gil-Monte, García-Juesas y Figueiredo-Ferraz (2010), quienes en un estudio de dos seguimientos con una separación de un año, descubrieron un aumento significativo en los niveles de agotamiento emocional, pero no hubo cambios significativos en los niveles de realización personal 0 despersonalización. En el estudio de Gil-Monte y García Juesas (2008), observaron descenso en la realización personal frente al trabajo, y aumento del agotamiento y la despersonalización.

Más de un año: Se localizaron dos estudios, el primero de Deary, Watson y Hogston (2003), quienes realizaron un seguimiento en varios momentos (en el mes 12, 24, y al final de la carrera) a universitarios desde el ingreso a su programa académico, y encontraron que estos experimentaron aumento del estrés en la medida que el programa avanzaba, así como aumento de la morbilidad psicológica. El segundo estudio es de Pantaleoni, Augustine, Sourkes y Bachrach (2014), quienes examinaron el síndrome de burnout en seis momentos - entre febrero de 2010 y febrero de 2012 - en residentes de pediatría: al inicio de la residencia, a mediados del primer año y fin del primer año, así como al inicio, mediados y fin del tercer año. Descubrieron cambios del "burnout" entre el inicio de la residencia y a mediados del primer año. Se halló que el agotamiento emocional aumentó, pero la despersonalización y la realización personal disminuyeron.

Como se observa, los datos son dispersos en la mirada longitudinal del síndrome, y este diseño es relevante para una aproximación a su configuración, adicionalmente, en la revisión de la literatura no se observan estudios bibliométricos-descriptivos del burnout a nivel longitudinal que analicen su desarrollo en el tiempo, por tanto, se considera que esta revisión puede ser importante para la comunidad científica al ser una aproximación al estado de la investigación longitudinal, al identificar un campo oportuno para activar, pero aún más, una línea necesaria para fortalecer los procesos de intervención. De allí que el objetivo de esta propuesta es realizar una revisión del estado del arte, tomando como referencia estudios con diseños longitudinales publicados entre el 2010 y el 2016.

\section{Método}

A través de un estudio bibliométrico, descriptivo-retrospectivo y de enfoque cuantitativo, se seleccionaron tres bases de datos que contienen estudios focalizados en psicología y ciencias de la salud: Science Direct, Scopus, Pub Med. Se describen variables como: nombres de revistas científicas, autores, tipo de acceso, año de publicación, país del 
Palacio-Sañudo et al - Una revisión de diseño longitudinales para estudiar el burnout

origen de la muestra, instrumentos y principales resultados.

Inicialmente se revisaron investigaciones sobre "burnout" 1983-2016, para luego delimitar al estudio del burnout académico (1983-2016 / 2010-2016), y focalizar estudios longitudinales realizados (2010-2016). Para esto, los términos claves de búsqueda fueron academic, burnout, longitudinal. El proceso de filtrado o inclusión de los artículos fue realizado en marzo de 2016. Ver gráfico 1. Los criterios para la inclusión de los artículos en el análisis fueron: a) estar en revistas indexadas en el Institute For Scientifc Information;

b) estar en revistas con multiplicidad de disciplinas;

c) estar en revistas con ISSN;

e) ser artículos de acceso abierto;

f) haberse realizado con un diseño longitudinal, independiente del número de medidas repetidas;

g) el diseño longitudinal se focalizaba en el estudio del "burnout" en estudiantes.

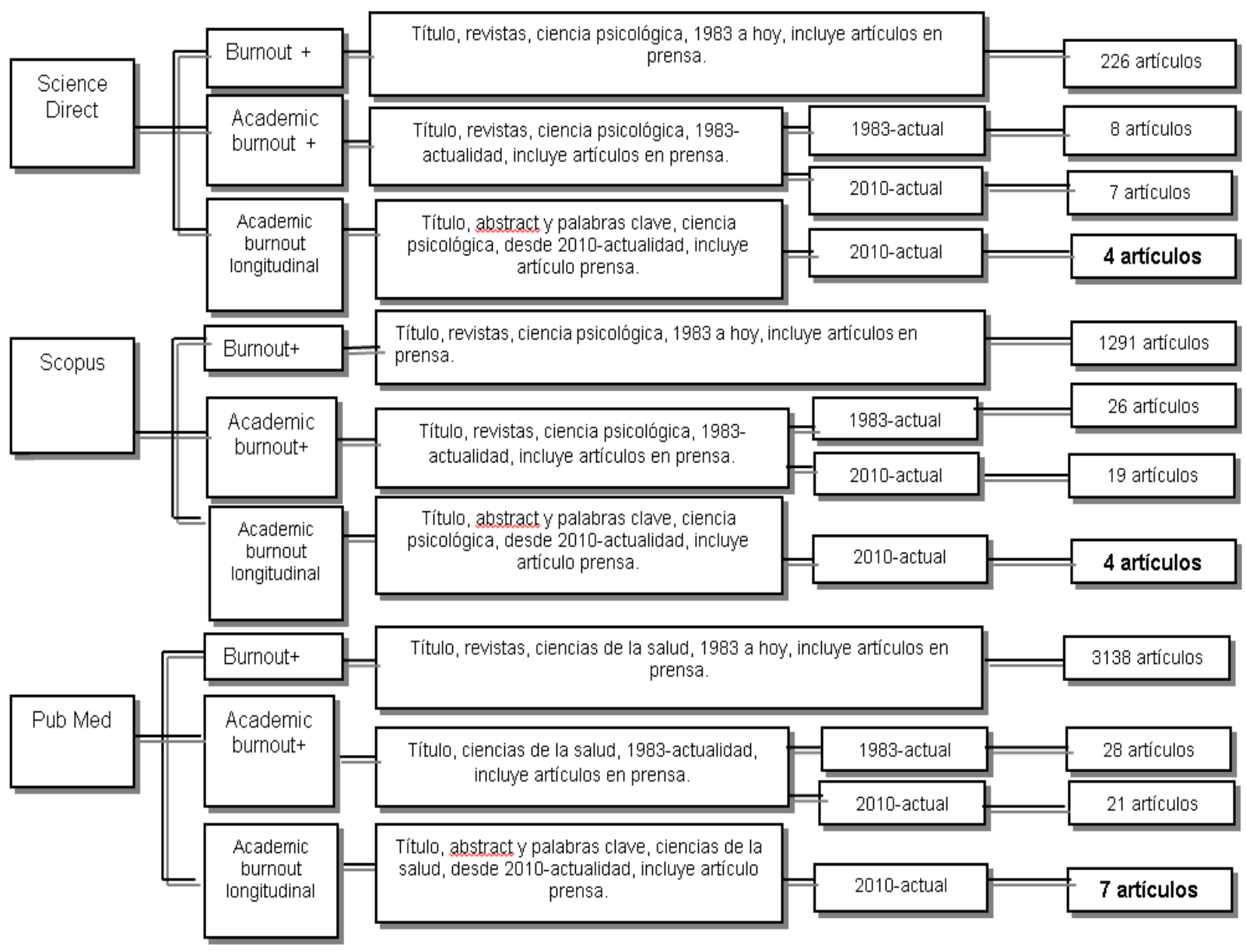

Gráfico 1. Rastreo del estudio longitudinal sobre "burnout" académico 


\section{Resultados}

El material analizado proviene de un universo de 4.655 artículos, de los cuales se identifican 15 investigaciones con diseño longitudinal para conocer el burnout académico en estudiantes: 4 artículos en Science Direct, 4 artículos en Scopus, 7 artículos en Pub Med. El número encontrado en Pub Med se asemeja al examinar dos bases de datos para las ciencias sociales: Science Direct y Scopus. El 50\% de los artículos son de acceso abierto para el público.

De las revistas reconocidas MedTeach presenta la mayor frecuencia de publicaciones en el estudio longitudinal del "burnout" académico. Se evidencia en la mayoría de las publicaciones autores en común, marcando la colaboración de investigadores en este campo de estudio:

Grupo 1: Tartas, Walkiewicz, Budzinski, Majkowicz \& Wojcikiewicz (2014), Walkiewicz, Tartas, Majkowicz \& Budzinski (2012), Tartas, Walkiewicz, Majkowicz \& Budzinski (2011).
Grupo 2: Noh, Shin \& Lee (2013), Noh, Chang, Jang, Lee \& Lee (2015), Chun, Park, Lee \& Kim (2014), Kim, Lee, Kim, Choi \& Lee (2015).

Grupo 3: Salmela-Aroa \& Tynkkynenb (2012), Wang, Chow, Hofkens, \& Salmela-Aro (2015), Bask \& SalmelaAro (2013).

Grupo 4: Galbraith \& Merrill (2012, 2015).

Grupo 5: diversos autores como Young, Fang, Golshan, Moutier \& Zisook (2012), Ying, Wang, Lin, \& Chen (2016). Ver tabla 1.

En los 15 artículos relacionados con el estudio longitudinal del "burnout" académico, se observa un aumento en publicaciones de artículos desde el 2011 al 2015, fluctuante para 2014, pero con una tendencia de 4 artículos por año. Por otra parte, sigue siendo de interés la investigación longitudinal del "burnout" académico, identificándose un artículo en el 2016 (Ver gráfico 2).

Tabla 1. Revista, autores y acceso a las publicaciones en el estudio longitudinal del "burnout" académico 2010-actualidad.

\begin{tabular}{|c|c|c|c|}
\hline Base de datos & Revista & Autores & Acceso \\
\hline \multirow{4}{*}{ Science Direct } & Learning and Individual Differences & Noh, Shin \& Lee. & \\
\hline & Journal of Adolescence & Salmela-Aroa \& Tynkkynenb. & \\
\hline & Learning and Instruction & Wang, Chow, Hofkens \&Salmela-Aro. & Open Access \\
\hline & Personality and Individual Differences & Ying, Wang, Lin, \& Chen. & \\
\hline \multirow{4}{*}{ Scopus } & Journal of Further and Higher Education & Galbraith \&Merrill. & \\
\hline & Europ. Jour. of Psychology of Education & Bask \& Salmela-Aro. & \\
\hline & Journal of College Student Development & Galbraith \& Merrill. & No open Acces \\
\hline & Academic Psychiatry & Young, Fang, Golshan, Moutier \& Zisook. & \\
\hline \multirow{7}{*}{ PubMed } & BMC MedEduc. & Tartas, Walkiewicz, Budzinski, Majkowicz \& Wojcikiewicz. & Open Acces \\
\hline & J ReligHealth. & Noh, Chang, Jang, Lee \& Lee. & No open Acces \\
\hline & J SurgEduc & Lindeman, Sacks, Hirose \& Lipsett. & No open Acces \\
\hline & Korean J Med. Educ. & Chun, Park, Lee \& Kim. & Open Acces \\
\hline & MedTeach & Walkiewicz, Tartas, Majkowicz \& Budzinski. & No open Acces \\
\hline & MedTeach & Tartas, Walkiewicz, Majkowicz \& Budzinski. & Open Acces \\
\hline & Stress Health & Kim, Lee, Kim, Choi \& Lee. & No open Acces \\
\hline
\end{tabular}


Palacio-Sañudo et al - Una revisión de diseño longitudinales para estudiar el burnout

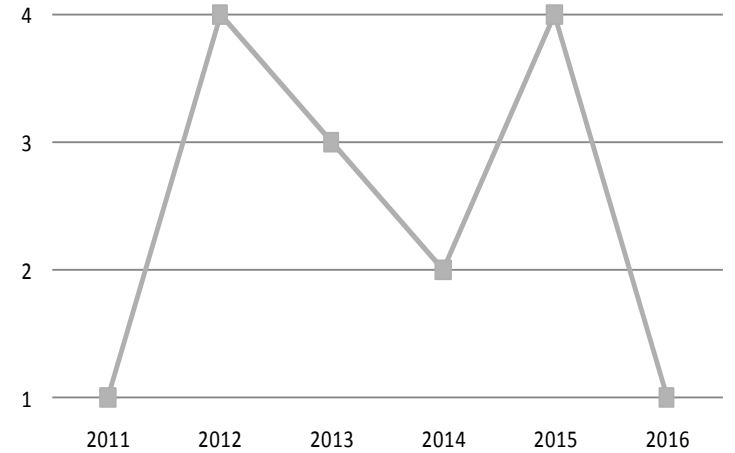

Gráfico 2. Número de artículos longitudinales por año

Fuente: Elaboración propia

De acuerdo con los artículos registrados en acceso abierto desde 2011 al 2015, los estudios longitudinales sobre "burnout" académico se han realizado sobre todo en tres continentes: Asia (Korea, Finlandia, China), Europa (Polonia) y América del Norte (Estados Unidos) pero con mayor frecuencia en países como Korea y Estados Unidos. Ver gráfico 3.

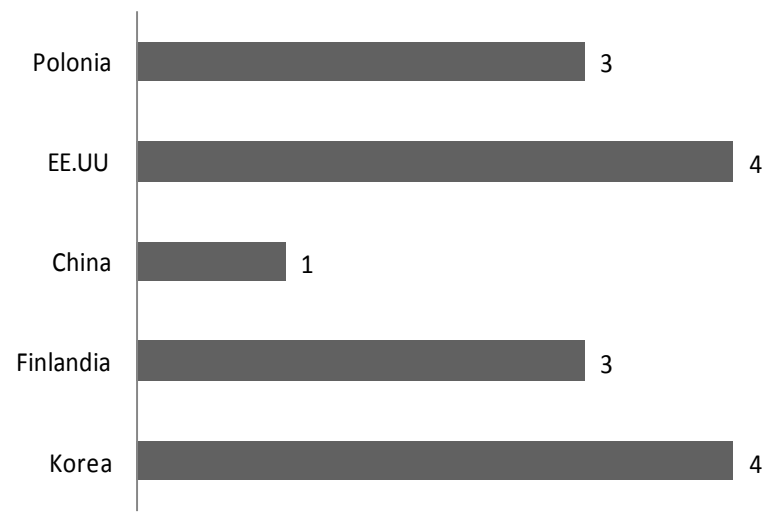

Gráfico 3. Número de artículos de acuerdo al origen de la muestra.

Fuente: Elaboración propia

Con respecto a la revisión de cada resultado, en las investigaciones se confirma la inestabilidad del síndrome. Primeramente, se encontró que los estudios longitudinales se enfocan en muestras de secundaria y universitaria, en este sentido existe una diversidad en instrumentos de medida y adaptaciones de acuerdo con el contexto cultural: Versión Coreana del MBI-SS, Inventario de Burnout de escuela, Academic Burnout Inventory, Manslach's Burnout Inventory (MBI). Ver tabla 2.

Los estudios identificados muestran que el rastreo del "burnout" académico se ha realizado en 3 y 4 seguimientos, tratando de identificar la evolución de sus niveles en los sujetos, pero se encuentra una gran variabilidad (aumento o descenso) de su severidad a lo largo del tiempo:

Noh et al. (2013), registraron que el cansancio emocional y cinismo progresan del t1 al t3, en su opuesto, la ineficacia es mayor en $t 1$, desciende en t2, pero en t3 supera el puntaje de t1. Salmela-Aroa y Tynkkynenb (2012), reconocieron en chicos de secundaria que el "burnout" presenta una estabilidad en $t 1-t 2$, e inicia su incremento en t3-t4; en el caso de las chicas de secundaria, el estado de desgaste aumenta desde el t1 al t4; el "burnout" en chicos y chicas de vocacional, después de $t 1$ presenta descensos hasta el t4.Wang et al. (2015), señalan aumento del "burnout" desde el t1 al t3. Ying et al. (2016) indican aumento del "burnout" del t1 al t3; Tartas et al. (2014), identificaron un descenso progresivo del burnout desde t1 a t3; Chun et al. (2014), reportan en agotamiento, aumento en un t2 y descenso para el t3; en el caso del cinismo e ineficacia, disminución del t1 al t3. Tartas, et al. (2011), informan los factores asociados, pero no reportan el comportamiento del "burnout" en cada medición.

\section{Conclusiones}

La exploración permitió encontrar un número de estudios originales en bases de datos como PubMed, casi en igual medida que en Science Direct y Scopus. Se observa la aceptación del "burnout" como una entidad independiente e insidiosa relacionada con problemáticas 
Revista Búsqueda - Enero/Junio de 2017

Tabla 2. Características de estudios longitudinales en Open Acces sobre "burnout" académico

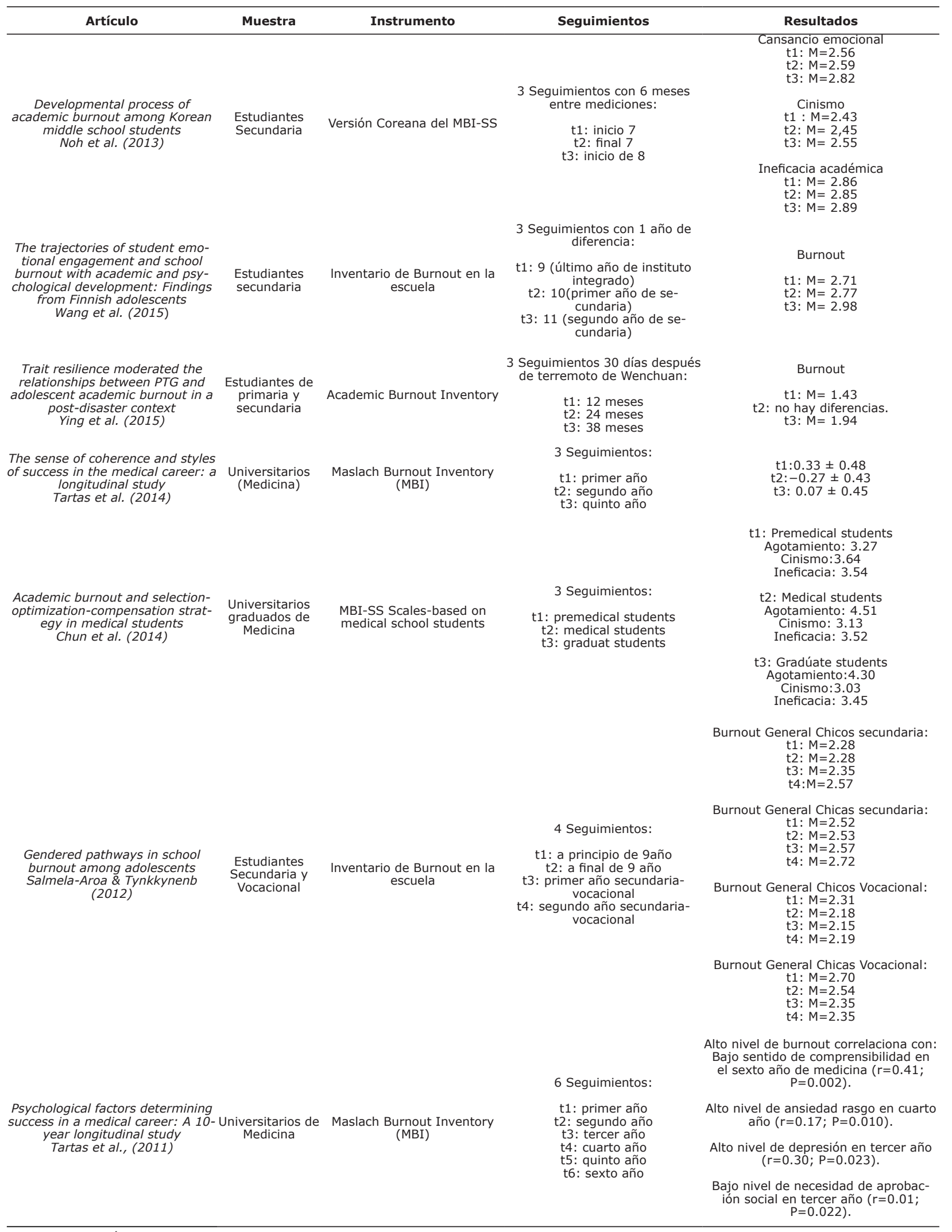

Fuente: Elaboración propia 
de salud física-mental (Aluja, 1997; Tang, Wing-Tung, Schwarzer y Schmitz, 2001; Grazziano y Ferraz, 2010; Yussuf, Issa, Ajiboye y Buhari, 2013). Además la configuración de sus síntomas cognitivos, emocionales, sociales y físicos afectan la salud en general, pero su incidencia se ha estudiado principalmente en médicos, enfermeras, psiquiatras o profesionales directamente relacionados con la atención y el cuidado de pacientes. Su tratamiento como síndrome, aunque no se incluye en manuales de diagnósticos psiquiátricos, evidencian que el estudio del "burnout" requiere una perspectiva multidisciplinar, convirtiéndose de interés, en un primer momento, para médicos, enfermeros, psicólogos, psiquiatras y odontólogos, y por consiguiente se ha focalizado en las revistas de estos campos.

Del mismo modo, se identificó que el estudio del "burnout" académico a través de diseños longitudinales en los últimos 5 años se ha efectuado en lugares específicos como Asía, África, Europa y América del Norte. Estos estudios son en gran medida relacionados con el síndrome en secundaria, y su estudio parece ser dependiente de cada zona geográfica.

Estudiar el burnout en secundaria parece ser una tendencia en investigación en los últimos 5 años, aunque no ha sido estudiado en Latinoamérica en este nivel de educación, por las particularidades de los sistemas educativos, al parecer por no ser lo suficientemente demandantes para generar un estado de agotamiento, cinismo e ineficacia ante el trabajo académico. No obstante, a pesar de ser poco probable considerar el burnout académico fuera de contextos universitarios en Latinoamérica, su futura exploración en niños y adolescentes obliga también a la adaptación o construcción de instrumentos puntuales tal como se demuestra en otros contextos; Ante esta exploración debe tenerse precaución en el diagnóstico, porque su expresión sintomática cognitiva, emocional, socialfamiliar y somática podría confundirse con la manifestación de otras entidades como la ansiedad y depresión (Caballero, Gutiérrez, y Palacio, 2015). De todos modos, no es nuevo el planteamiento del burnout como una entidad presente en niños y adolescentes (Garcés de los Fayos, 1995).

Este estado negativo se presenta como resultado de un proceso en el que sus características no emergen de manera súbita, sino de forma progresiva (Álvarez Gallego y Fernández Ríos, 1991). Sin embargo, su configuración imprevista, no solo está relacionada con los estresores académicos, sino también con la presencia intensa y persistente de los factores mencionados anteriormente como riesgos psicosociales: factores personales, sociales, económicos y familiares (Aceves, López, Moreno, Jiménez, y Campos, 2006). Es un estado aunque evolutivo, fluctuante (Chernis, 1980; Mafla, Masbuscay y Ortiz, 2009) y contagioso en el tiempo (Maslach, 2009), puede descender o incrementarse, dependiendo de la presencia de los factores de riesgo.

Los artículos identificados en el rastreo entre 2010-2016 sobre el estudio longitudinal del burnout presentan una metodología diferencial y resultados muy diversos. En algunos de estos se observan limitaciones por la cercanía entre las observaciones, la heterogeneidad de los instrumentos de medida del burnout, además de la evaluación fuera del contexto académico - población víctima de desastres naturales. No obstante, se destacan fortalezas como la utilización de análisis multivariados y muestras con tamaños superiores a 200 sujetos en cada medición. Aunque existan pérdidas importantes de participantes entre una medición y otra, se destaca que las limitaciones de los estudios longitudinales se equilibran con los costos, esfuerzos y riesgos que implica valorar un número importante de sujetos en contextos académicos en varios tiempos. 
Al respectodela configuración delburnout académico, los estudios rastreados evidencian la evolución del síndrome en el tiempo, que puede explicarse por la permanencia, intensidad y cantidad de estresores que rodean al sujeto durante determinado tiempo de actividades académicas. Esta presencia prolongada de estresores generan síntomas propios del agotamiento, cinismo e ineficacia, y se configura una respuesta a las condiciones prolongadas de estrés crónico (Ortega y López, 2004; Suárez, Caballero y Palacio, 2013).

Los estudios confirman estos planteamientos sobre la oscilación del síndrome. En algunas investigaciones los estudiantes atraviesan por el desarrollo del burnout (Noh et al., 2013; Wang et al., 2015; Ying et al, 2015; Chun et al, 2014; Salmela-Aroa y Tynkkynenb, 2012), mientras otros experimentan una disminución en algunas de sus dimensiones (Tartas et al, 2014). En los estudios longitudinales se demuestra un descenso de la afección luego de recesos, pero otros estudios reflejan la estabilidad en el patrón de afección. Además, sugieren que el síndrome evoluciona en severidad a lo largo del tiempo, desligándose de la estabilidad, por lo cual el síndrome de burnout es un estado fluctuante de carácter psicosocial con repercusión temporal en la salud mental. Esta tendencia es coherente con Mafla, et al., (2009), quien estima que el burnout es una condición humana producto del acumulado de estresores, pero probablemente sería una situación esporádica, y por tanto, posible para reducirse o no aparecer.

Este constructo psicológico también se ha catalogado como un fenómeno secuencial en el origen de las dimensiones, sin embargo, en esta línea se encontraron divergencias en el orden de aparición del agotamiento, cinismo e ineficacia, o bien al momento de establecer los componentes centrales que explican el síndrome del desgaste (Guerrero y Vicente, 2001; Llorens, et al, 2005; Salanova, et al., 2005). Según Salanova \& Llorens (2008), el orden en el desarrollo de las dimensiones del burnout estaría determinado por la ocupación, estimando la existencia de dos procesos:

- Primero, aquel propio de las profesiones de ayuda en el que, el individuo se consume emocionalmente y luego se distancia.

- El segundo, basado en la pérdida de confianza en las competencias. Este último sería más relevante en el burnout académico.

En este sentido, no se podría especificar el orden de aparición del agotamiento, cinismo e ineficacia en estudiantes, ni la dirección del curso longitudinal del síndrome, pero se puede estimar que un sujeto con dificultades en recursos personales $y / 0$ inmerso en factores de riesgo del contexto social-familiarorganizacional, sería recidivante, de modo que la recuperación podría ser inter-episódica, dando paso a recaídas.

Ante esta problemática existen diversas alternativas de intervención, desde estrategias individuales (técnicas fisiológicas, técnicas cognitivas, técnicas conductuales, técnicas sociales) y estrategias organizacionales (Guerrero y Rubio, 2005). Las primeras, orientadas a fortalecer los recursos personales, y las segundas, centradas en equilibrar las demandas y recursos del contexto (Salanova y Llorens, 2008). Los estudios documentan la facilidad para la reducción del agotamiento a través de ejercicios de relajación, pero encuentran resistencia a disolver el cinismo e ineficacia al no involucrar las fortalezas personales de los individuos; del mismo modo la intervención ha sido poco enfocada en la organización o institución donde se labora o estudia, en consecuencia, son necesarios los programas centrados en el fortalecimiento de los recursos psicológicos y programas centrados en la disminución de los factores de riesgo 
Palacio-Sañudo et al - Una revisión de diseño longitudinales para estudiar el burnout

organizacionales para consolidarlas como organizaciones integralmente saludables (Shaufeli, 2003; Salanova y Llorens, 2008).

Aunque la aplicación de estas estrategias depende del diagnóstico, pueden existir restricciones para intervenir las organizaciones en función de sus demandas-recursos, de modo que, en estos casos,la intervención inicial de burnout generalmente podría enfocarse en estrategias individuales:

1) El desarrollo de estrategias de regulación emocional (Cabanach, Souto, Fernández-Cervantes y Freire, 2011), para la intervención de los pensamientos de ineficacia;

2) La vinculación a redes de apoyo efectivas (Moral, González \& Landero, 2011), esencial para el fomento del trabajo en equipo con el fin de actuar sobre el cinismo, apatía o desinterés por el trabajo académico;

3) Programas de actividad física en sesiones bien definidas para intervenir sobre el agotamiento o cansancio (Gerber, et al., 2013).

La intervención del burnout podría ser directamente beneficiaria de los estudios de seguimiento con varios cortes de evaluación (al menos 4 durante dos años continuos), para clarificar el patrón estacional y el curso del burnout, de manera a responder: ¿cuál es la trayectoria del burnout durante un semestre académico y el orden de su configuración tridimensional (agotamiento, cinismo, ineficacia)?, ¿cuál es el espacio temporal para el establecimiento del burnout en su mayor nivel de severidad desde su configuración tridimensional?, ¿cuál es la temporalidad del patrón de las dimensiones del burnout - estático o fluctuante?, y ¿qué tan indisoluble o severo es el burnout durante un semestre académico o durante la carrera universitaria?

Los retos de la investigación para comprender la configuración del burnout académico apuntan a los estudios longitudinales y cualitativos, que permitan reconocer el origen y evolución del síndrome en universitarios, dados los factores del contexto organizacional -educativo, social-familiar y los recursos personales del sujeto. Además, se requiere la realización de estudios epidemiológicos que estimen la incidencia y prevalencia del burnout con instrumentos de medición reformados para identificar mejor la presencia de casos clínicos.

Por otro lado, la comparación con estudios transculturales permitiría comprender la incidencia de la cultura en el desarrollo del burnout, más allá de las diferencias de medias entre población de otros países (Schaufeli, Martínez, Marqués-Pinto, Salanova y Bakker, 2002; Golembiewski, Scherb y Boudreau, 1993; Schaufeli, 2003; Riolli y Savicki, 2003).

Es importante destacar que otros retos de la investigación deben direccionarse a una conceptualización acerca de cómo se configura el burnout en su cualidad y en el tiempo, además de continuar mejorando la delimitación conceptual de su naturaleza, para evitar los falsos positivos. Para esto es indispensable mejorar los instrumentos de evaluación objetivos, teniendo en cuenta que la diversidad de criterios en los puntos de corte dificulta el diagnóstico sobre la incidencia o prevalencia del síndrome.

Además, el sentido positivo de las preguntas en la dimensión eficacia/ realización personal difieren de las dimensiones de agotamiento y cinismo/ despersonalización, al estar formuladas en negativo, lo cual afecta la sensibilidad de estos instrumentos (Caballero, et al, 2010), y esto se observa en los instrumentos de medición como el Maslach Burnout Inventory (MBI) de Maslach y Jackson (1981), Maslach Burnout Inventory MBI-GS (General Survey) de Schaufeli, Leiter, Maslach y 
Jackson (1996), y el Maslach Burnout / inventory-Student Survey (MB/-SS) de Schaufeli, et al (2002).

Finalmente, si las universidades desean consolidarse como organizaciones saludables deben priorizar la promoción de la salud-calidad de vida, prevención de los riesgos y vigilancia en salud de sus estudiantes-trabajadores, con el fin de fortalecer el desarrollo humano e incrementar la calidad de vida, y a su vez, podrían ser promotoras de la salud en sus familias, en sus futuros ambientes laborales y la sociedad en general (Lange y Vio, 2006). En Colombia, los adolescentes-jóvenes universitarios presentan un número importante de problemas relacionados con su salud, entre los cuales se incluye el síndrome de "burnout", el cual puede - además - incrementa la severidad de psicopatologías subyacentes, de allí que las universidades que logren conformarse como organizaciones saludables podrán ser una alternativa para la contingencia o mejora de los problemas de salud de sus estudiantes.

\section{Referencias}

Aceves, G.A.G., López, M.Á.C., Moreno, S., Jiménez, F.F.S. \& Campos, J.D.J. S. (2006). Síndrome de burnout. Arch Neurocien (Mex), 11(4), 305-309.

Aluja, A. (1997). Burnout profesional en maestros y su relación con indicadores de salud mental. Boletín de Psicología, 55, 47-61.

Álvarez Gallego, E. y Fernández Ríos, L. (1991). El Síndrome de" Burnout" o el desgaste profesional. Revista de la Asociación Española de Neuropsiquiatría, 11(39), 257-265.

Bask, M. y Salmela-Aro, K. (2013). Burned out to drop out: Exploring the relationship between school burnout and school dropout. European journal of psychology of education. 28(2), 511-28.

Bresó, E. y Salanova, M. (2005). Efectos significativos del uso de las creencias de ineficacia como componente del burnout académico en estudiantes universitarios. Jornades de Foment de la Investigació. Recuperado de http://www.uji.es/bin/publ/edicions/ jfi8/psi/32.pdf.
Bresó, E. (2008). Well-bing and performance in academic sttings: the prediting role of self-efficacy. Disertación doctoral no publicada. Universidad Jaume I de Castellón, Castellón de la Plana.

Caballero, C. y Bresó, E. (2015). Burnout in university students. Psicología desde el caribe, 32 (3), 32(3), 424441. doi: 10.14482 psdc.32.3.6217

Caballero, C., Palacio, J. y Hederich, C. (2012). El burnout académico: Prevalencia y factores asociados en estudiantes universitarios del área de la salud de la ciudad de Barranquilla. Tesis doctoral. Universidad del Norte.

Caballero, C., Gutiérrez, O. y Palacio, J. (2015). Relación del burnout $y$ el engagement con depresión, ansiedad y rendimiento académico en estudiantes universitarios. Salud Uninorte, 31(1), 59-69.

Cabanach, R.G., Gestal, A.J.S., Cervantes, R.F. y Rodríguez, C.F. (2011). Regulación emocional y burnout académico en estudiantes universitarios de Fisioterapia. Revista de Investigación en Educación, 2(9), 7-18. 
Palacio-Sañudo et al - Una revisión de diseño longitudinales para estudiar el burnout

Cherniss, C. (1980). Professional burnout in human service organizations. New York: Praeger.

Chun, KH., Park, YS., Lee, YH. y Kim, SY. (2014). [Academic burnout and selection-optimization-compensation strategy in medical students]. Korean J MedEdu, 26(4), 299-308. doi: 10.3946/kjme.2014.26.4.299.

Deary, I.J., Watson, R. y Hogston, R. (2003). A longitudinal cohort study of burnout and attrition in nursing students. Journal of Advanced Nursing, 43, 71-81.

Galbraith, C.S. y Merrill, G.B. (2012). Academic and work-related burnout: A longitudinal study of working undergraduate university business students. Journal of College Student Development, 53(3), 453-463.

Galbraith, C.S. y Merrill, G.B. (2015). Academic performance and burnout: An efficient frontier analysis of resource use efficiency among employed university students. Journal of further and Higher Education, $39(2)$, 255-277. doi:10.1080/03098 77X.2013.858673

Garcés de Los Fayos, E.J.G. (1995). Burnout en deportistas. Revista de psicología social aplicada, 3, 5-16.

Gerber, M., Lang, C., Feldmeth, A.K., Elliot, C., Brand, S., Holsboer-Trachsler, E. y Pühse, U. (2013). Burnout and Mental Health in Swiss Vocational Students: The Moderating Role of Physical Activity.

Gil-Monte, P.R. y García-Juesas, J.A. (2008). Efectos de la sobrecarga laboral y la autoeficacia sobre el síndrome de quemarse por el trabajo (burnout). Un estudio longitudinal en enfermería. Revista Mexicana de Psicología, 25(2):329-337.
Barona, E.G., y Jiménez, J.C.R. (2005). Estrategias de prevención e intervención del Burnout en el ámbito educativo. Salud Mental, 28(5), 27-33.

Golembiewski, R.T., Scherb, K. y Boudreau, R.A. (1993). Burnout in cross-national settings: Generic and model-specific perspectives. Professional burnout: Recent developments in theory and research, 217-236.

Grau-Alberola, E., Gil-Monte, P.R., GarcíaJuesas, J.A. y Figueiredo-Ferraz, $\mathrm{H}$. (2010). Incidence of burnout in Spanish nursing professionals: A longitudinal study. International Journal of Nursing Studies, 47(8), 1013-1020.

Grazziano, E.S. y Ferraz Bianchi, E.R. (2010). Impacto del estrés ocupacional y burnout en enfermeros. Enfermería Global, 18, 1-20.

Guerrero, E. y Vicente, F. (2001). Síndrome de burnout o desgaste profesional y afrontamiento del estrés en el profesorado. Cáceres: Servicio de Publicaciones de la Universidad de Extremadura.

Kim, B., Lee, M., Kim K., Choi, H. y Lee, SM. (2015). Longitudinal Analysis of Academic Burnout in Korean Middle School Students. Stress Health, 31(4) 281-289. doi: 10.1002/ smi. 2553.

Llorens, S., García-Renedo, M. y Salanova, M. (2005). Burnout como consecuencia de una crisis de eficacia: un estudio longitudinal en profesores de secundaria. Revista de Psicología del Trabajo y de las Organizaciones, 21:55-70.

Lange, L. y Vio, F. (2006). Guía para universidades saludables. Chile: OPS, Pontifica Universidad Católica, otros. 
Mafla, A., Masbuscay, M. y Ortiz, A. (2009). Síndrome de burnout en estudiantes de odontología: 18 meses de seguimiento. Revista Universidad y Salud, 1 (11), 52-70.

Maslach, C. (2009). Comprendiendo el Burnout. Ciencia y Trabajo, 11(32), 37-43.

Maslach, C. y Jackson, S. E. (1981). Maslach Burnout Inventory. Palo Alto, California: Consulting Psychological Press.

Moral, J., González, M. T. y Landero, R. (2011). Tratamiento de la fibromialgia en mujeres y evaluación del burnout en amas de casa. Monterrey, NL México.

Noh, H., Shin, H. y Lee, S. M. (2013). Developmental process of academic burnout among Korean middle school students. Learning and Individual Differences, 28, 82-89. doi:/10.1016/j.lindif.2013.09.014

Noh, H., Chang, E., Jang, Y., Lee, JH. y Lee, SM. (2015). Suppressor Effects of Positive and Negative Religious Coping on Academic Burnout Among Korean Middle School Students. J Relig Health, 55(1), 135-146.

Ortega, C. y López, F. (2004). El burnout o síndrome de estar quemado en los profesionales sanitarios: revisión y perspectivas. International Journal of Clinical and Health Psychology, 4(1), 137-60.

Pantaleoni, J. L., Augustine, E. M., Sourkes, B. M. y Bachrach, L. K. (2014). Burnout in Pediatric Residents Over a 2-Year Period: A Longitudinal Study. Academic pediatrics, 14(2), 167-172.

Park, Y. M. y Lee, S. M. (2013). A longitudinal analysis of burnout in middle and high School Korean teachers. Stress and Health, 29(5), 427-431.
Párraga, J. (2005). Eficacia del programa I.R.I.S. para reducir el síndrome de burnout y mejorar las disfunciones emocionales en profesionales sanitarios. [Disertación doctoral]. Universidad Extremadura, Extremadura, España.

Riolli, L. \& Savicki, V. (2003). Optimism and coping as moderators of the relation between work resources and burnout in information service workers. International Journal of Stress Management, 10(3):235-252.

Salanova, M., Martínez I. M. y Lorente, L. (2005). ¿Cómo se relacionan los obstáculos y facilitadores organizacionales con el burnout docente?: Un estudio longitudinal. Revista de Psicología del Trabajo y de las Organizaciones, 21 (1-2), 37-54.

Salanova, M. y Llorens, S. (2008). Estado actual y retos futuros en el estudio del burnout. Papeles del Psicólogo, 29 (1), 59-67.

Salanova, M., Grau, R., Martínez, I. y Llorens, S. (2004). Facilitadores, obstáculos, rendimiento académico relacionado con la satisfacción con los asuntos del estudio. (Periódico). El País Universal.

Salanova, M., Martínez, I. M. y Lorente, L. (2005). ¿Cómo se relacionan los obstáculos y facilitadores organizacionales con el burnout docente?: Un estudio longitudinal. Revista de Psicología del Trabajo y de las Organizaciones, 21(1-2):37-54.

Salmela-Aroa, K., y Tynkkynen, L. (2012). Gendered pathways in school burnout among adolescents. Journal of Adolescence, 35(4), 929-939. doi:10.1016/j.adolescence.2012.01.001

Schaufeli, W. (2003). Past perfomance and future perspectives of burnout research. Journal of industrial Psychology, 29(4):1-15. 
Palacio-Sañudo et al - Una revisión de diseño longitudinales para estudiar el burnout

Schaufeli, W. B., Martínez, I., MarquésPinto, A., Salanova, M. \& Bakker, A. (2002). Burnout and engagement in university students: A cross national study. Journal of Cross-Cultural Studies, 33, 464-481.

Schaufeli, W.B., Leiter, M.P., Maslach, C. y Jackson, S.E. (1996). The Maslach Burnout Inventory-General Survey. In C. Maslach, S.E. Jackson, \& M.P. Leiter (Eds.), Maslach Burnout Inventory. Palo Alto, CA: Consulting Psychologists Press.

Schaufeli, W.B., Salanova, M., GonzálezRomá, V. y Bakker, A.B. (2002). The measurement of engagement and burnout: A two sample confirmatory factor analytic approach. Journal of Happiness Studies, 3, 71-92.

Suárez, Y., Caballero, C. y Palacio, J. (2013). Factores psicosociales asociados al burnout académico en jóvenes de una Universidad Pública Colombiana. Proyecto Jóvenes Investigadores Colciencias.

Tang. S. C., Wing-Tung, Au., Schwarzer, R. y Schmitz, G. (2001). Mental health outcomes of job stress among chinese teachers: role of stress resource factors and burnout. Journal of Organizational Behavior, 22, 887901.

Tartas, M., Walkiewicz., Budzinski, W., Majkowicz, M. y Wojcikiewicz, K. (2014). The sense of coherence and styles of success in the medical career: a longitudinal study. Med Educ,28, 14, 254. doi: 10.1186/ s12909-014-0254-5.

Tartas, M., Walkiewicz, M., Majkowicz, M. y Budzinski, W. (2011). Psychological factors determining success in a medical career: a 10-year longitudinal study. MedTeach, 33(3),163-172. doi: 10.3109/0142159X.2011.544795
Wade, D. C., Cooley, E. y Savicki, V. (1986). A longitudinal study of burnout. Children and Youth Services Review, 8(2), 161-173.

Walkiewicz, M., Tartas, M., Majkowicz, M. y Budzinski, W. (2012). Academic achievement, depression and anxiety during medical education predict the styles of success in a medical career: a 10-year longitudinal study. Med Teach, 34(9), 611-619. doi: 10.3109/0142159X.2012.687478.

Wang, M.-T., Chow, A., Hofkens, T. y Salmela-Aro, K. (2015). The trajectories of student emotional engagement and school burnout with academic and psychological development: Findings from Finnish adolescents. Learning and Instruction, 36, 57-65. doi:/10.1016/j. learninstruc.2014.11.004

Yan, C. E. y Tang, S. C. (2003). The role of individual, interpersonal and organizational factors in mitigating burnout among elderly Chinese volunteers. International Journal of Geriatric Psychiatry, 18, 795-802.

Ying, L., Wang, Y., Lin, C. y Chen, C. (2016). Trait resilience moderated the relationships between PTG and adolescent academic burnout in a post-disaster context. Personality and Individual Differences, 90, 108-112. doi:10.1016/j.paid.2015.10.048

Young, C., Fang, D., Golshan, S., Moutier, C., y Zisook, S. (2012). Burnout in premedical undergraduate students. Academic Psychiatry, 36(1), 11-16. doi:10.1176/appi.ap. 10080125

Yussuf, AD., Issa, BA., Ajiboye, PO. y Buhari, OI. (2013). The correlates of stress, coping styles and psychiatric morbidity in the first year of medical education at a Nigerian University. Afr J Psychiatry (Johannesbg), 16(3), 206-215. doi: 10.4314/ajpsy. v16i3.28 